\title{
A Case of COPD with Left Endobronchial Hamartoma by Bronchoscopic Intervention
}

\author{
Yi Gong1,2, Lu Lư ${ }^{3}$, Feng Long1, Haihua Yang1, Xiaodong Chen ${ }^{2 *}$, Shengqing Li ${ }^{2}$ \\ ${ }^{1}$ Department of Respiratory Medicine, Huashan Hospital North, Fudan University, Shanghai, China \\ ${ }^{2}$ Department of Respiratory Disease, Huashan Hospital, Fudan University, Shanghai, China \\ ${ }^{3}$ Department of Pathology, Huashan Hospital North, Fudan University, Shanghai, China \\ Email: hallengong@sina.com, *xdchen8@hotmail.com
}

How to cite this paper: Gong, Y., Lu, L., Long, F., Yang, H.H., Chen, X.D. and Li, S.Q. (2018) A Case of COPD with Left Endobronchial Hamartoma by Bronchoscopic Intervention. Case Reports in Clinical Medicine, 7, 550-556.

https://doi.org/10.4236/crcm.2018.711049

Received: October 8, 2018

Accepted: November 6, 2018

Published: November 9, 2018

Copyright $\odot 2018$ by authors and Scientific Research Publishing Inc. This work is licensed under the Creative Commons Attribution International License (CC BY 4.0).

http://creativecommons.org/licenses/by/4.0/

\begin{abstract}
Objective: Exploration for chronic obstructive pulmonary disease with branched air lumen hamartoma by bronchoscoptic treatment. Material and Method: An old man was referred to our hospital with shortness of breath for many years. Clinical evidence emphysema on respiratory examination, lung function and chest X-ray gave rise to concern. He was diagnosed as COPD and treated with ICS + LABA. But the shortness of breath is still existed. Initial assessment by CT suggested a left lower lobe collapse. Then bronchoscope identified a solid abnormality in main left bronchus. The pathology showed a benign neoplasm. Because of intolerance of operation, the patient was treated by brochoscopic intervention. The endoscopic intervention included resection by electrosurgical snare, electrocautery, argon plasma coagulation (APC). Result: After intervention, the neoplasm was partly removed and the pathological result was endobrochial hematoma. After treatment, the patient's panting had taken a turn for the better. After one month later, the patient's lung function improved a lot. Conclusion: In conclusion, endobronchial hamartomas are one of benign neoplasms. It can cause persistent bronchial obstruction and recurrent pneumonias. In this case, invasive endoscopic treatment provides an excellent outcome.
\end{abstract}

\section{Keywords}

Endobronchial Hamartoma (EH), Bronchoscopic Intervention, COPD

\section{Background}

Lobe collapse in COPD occurs in the presence of airway obstruction, usually caused by lung cancer, viscous sputum or bronchial foreign body. However, the 
cases due to benign origin are easy to be misdiagnosed, especially benign endobronchial tumors. Pulmonary hamartoma described common benign lung tumor with an incidence of $0.025 \%-0.32 \%$ [1]. There are two clinical types as location: intraparenchymal or endobronchial. Parenchymal hamartomas are usually asymptomatic and presenting as solitary pulmonary nodule. Endobronchial hamartoma (EH) originate from large bronchus and grow into the lumen. Common symptoms include such as cough, dyspnea, wheezing [1] [2] [3]. It may cause misdiagnoses with other chronic airway disease, especially who has COPD. We here reported a COPD patient with left endobronchial hamartoma.

\section{Case Report}

A 75-year-old man was referred to our hospital due to shortness of breath for 2 year after activity. Clinical evidence of emphysema on respiratory examination, lung function test and chest $\mathrm{x}$-ray gave rise to significant clinical concern (Table 1). He was diagnosed as COPD at first and treated with ICS plus LABA according to GOLD. But the patient became progressively more unwell and failed to settle with medical therapy. On examination, he was afebrile and showed no abnormal physical findings, including breath sounds. Laboratory tests showed an elevated level of erythrocyte sedimentation rate (ESR) with no abnormality in serum tumor makers or other infection data. Chest computed tomography (CT) showed a localized consolidation in the left lower lobe collapse prior to the visit to our hospital (Figure 1(a), Figure 1(b)). We speculated there is an endobronchial lesion in the left bronchus, such as foreign matter, granuloma, or an endobronchial tumor. Subsequent flexible bronchoscopy was performed to investigate the endobronchus, identifing a solid abnormality totally occluding the main left bronchus (Figure 2(a), Figure 2(b)). The primary pathology finding was bronchial polyp (Figure 3(a), Figure 3(b)). Because of poor lung function, the patient was unable to tolerate operation. A decision was made to undertake interventional treatment by flexible bronchoscopy. During the bronchoscopy the lesion was cauterized with 20 Watt Argon Plasma Coagulation (ERBOTOM ICC 200 APC, Erbe, Germany) and removed with cryoextraction. A rigid APC probe, $2.3 \mathrm{~mm}$ in diameter and $50 \mathrm{~cm}$ length, was used. Most of the bronchus neoplasm was removed (Figure 4(a), Figure 4(b)). Obstructing endobronchial lesion was coming from the dorsal portion of left lower lobe. On re-evaluation immediately after the endobronchial therapy, the lower lobe bronchus was partly exposed (Figure 4(c)) and the upper lobe bronchus was found to be entirely patent (Figure 4(d)). The post intervention period was uneventful and the patient was discharged after two days. Pathological examination identified a tumor protruding into the bronchial lumen and partially obstructing the bronchus, which contained mature cartilage and fat tissue in the deeper layer, and was finally diagnosed as endobronchial chondroid hamartoma (Figure 5(a), Figure 5(b)). The patient has been followed, with Chest computed tomography and pulmonary function tests after one month. The patient felt shortness of breath symptoms 
Table 1. Lung function before hospitalization.

\begin{tabular}{cccc}
\hline Lung function & Predictive value & Actual Value & percent $\%$ \\
\hline VC(L) & 2.91 & 2.04 & 70 \\
FEV1(L) & 2.27 & 0.92 & 41 \\
FEV1/FVC (\%) & & 44.07 & \\
\hline
\end{tabular}

Bronchodilation test: negative after inhaled bronchodilator $15 \mathrm{~min}$, FEV1 was increased 11\% (about $100 \mathrm{ml}$ ).

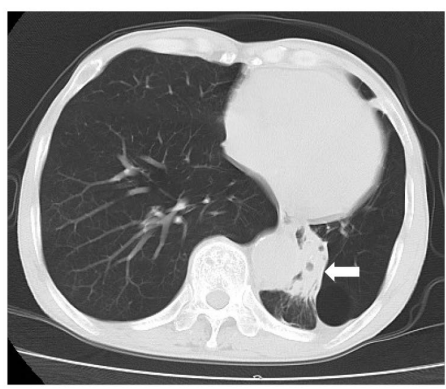

(a)

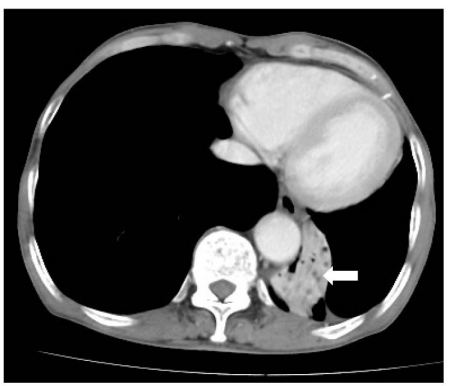

(b)

Figure 1. Chest CT scan ((a), (b)) showed a localized consolidation in the left lower lobe collapse. ((a) pulmonary window, (b) mediastinal window).

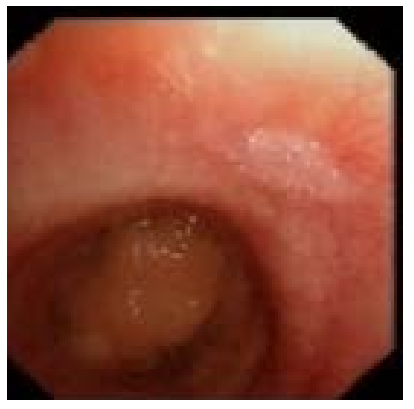

(a)

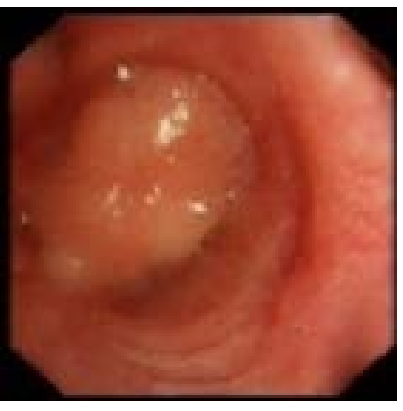

(b)

Figure 2. Bronchoscope examination showed an intra-bronchial tumor in the main left bronchus ((a), (b)). Main left bronchus was completely obstructed by a tumor with smooth surface, which is $2 \mathrm{~cm}$ from bronchial carina.

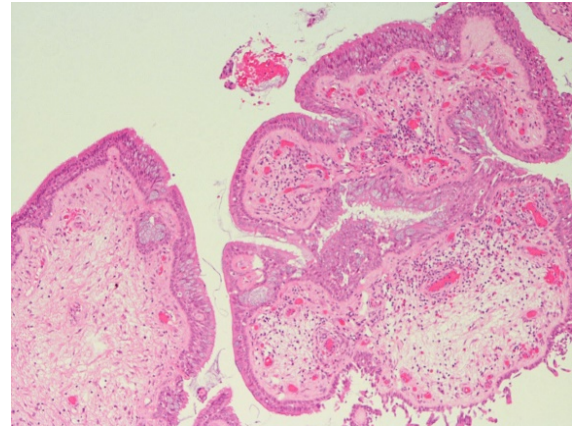

(a)

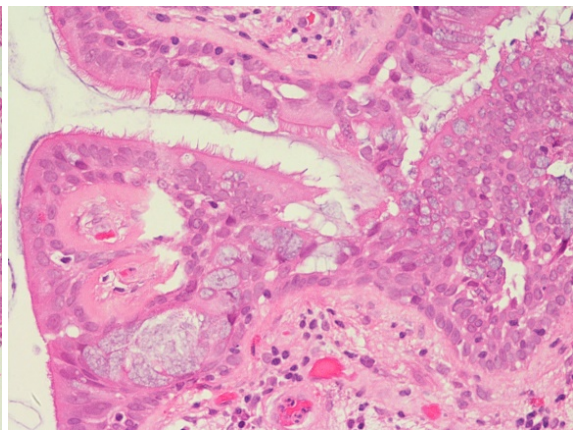

(b)

Figure 3. Histopathology of the tumor removed with bronchoscopy. After hematoxylin eosin staining, the polypoid tumor was constituted by amixture of fibrous connective tissue covered with ciliated columnar epithelium and squamous epithelial cells. (a) Low magnification view. (b) High magnification view. ((a) 10× HE; (b) 40× HE). 


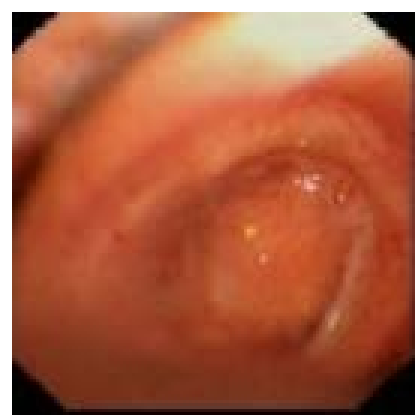

(a) before treatment

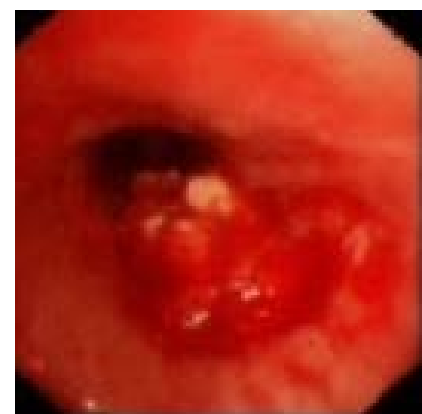

(c) the left lower lobe bronchus

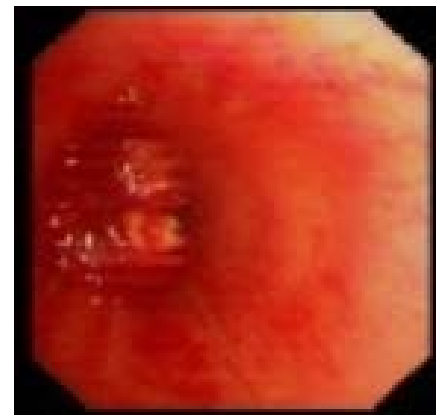

(b) on treatment

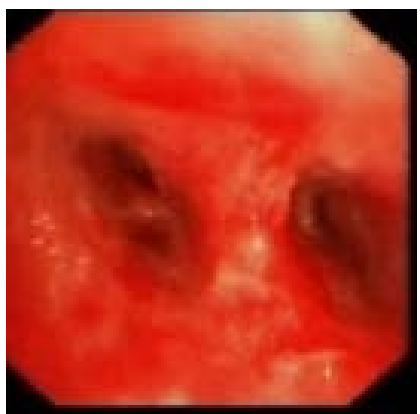

(d) the left upper lobe bronchus

Figure 4. Intervention treatment by fiberoptic bronchoscope with Argon Plasma Coagulator and cryoextraction ((a) before treatment, (b) after treatment). The recanalization of the lumen of the lower lobe bronchus was showed ((c), (d)).

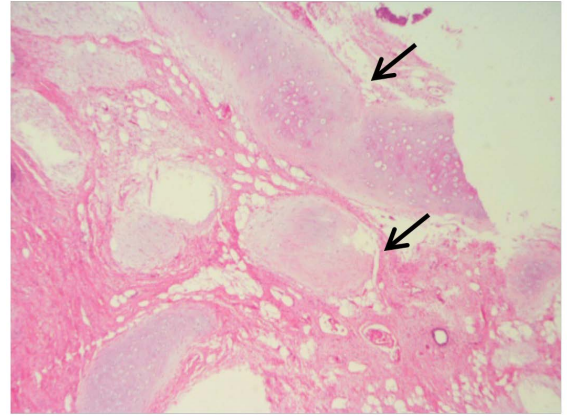

(a)

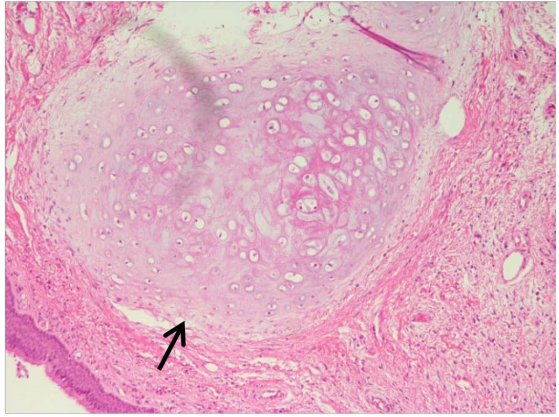

(b)

Figure 5. Histopathologic examination showing endobronchial hamartoma (hematoxylin and eosin, (a) $4 \times$, (b) 10×). Histopathology of the tumor removed with bronchoscopy shows cartilage component (arrow).

improved obviously, but chest CT scan still showed a localized consolidation in the dorsal portion of left lower lobe (Figure 6(a), Figure 6(b)). The patient's lung function improved a lot, FEV1 increased from $0.92 \mathrm{~L}$ to $1.55 \mathrm{~L}$ (Table 2).

\section{Discussion}

Both of bronchial fibroepithelial polyp and $\mathrm{EH}$ are benign tumors of the bronchus and are often difficult to differentiate based on clinical findings alone [4]. Benign lung tumors represent less than $1 \%$, and among these, hamartomas, with an incidence between $0.025 \%-0.32 \%$, are the most common. Bronchial fibroepithelial 


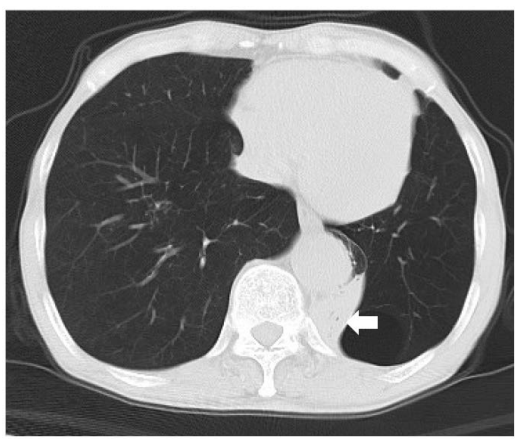

(a)

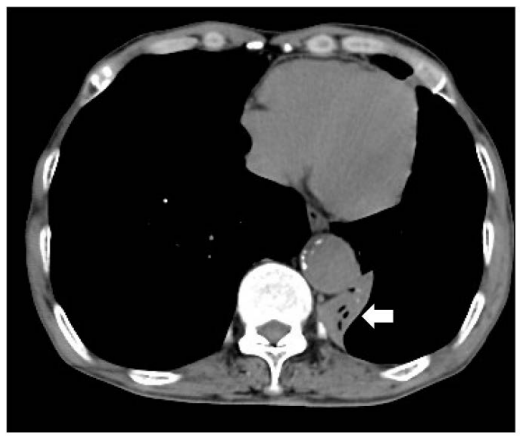

(b)

Figure 6. After one month of treatment, chest CT scan ((a), (b)) showed a localized consolidation in the dorsal portion of left lower lobe. ((a) Pulmonary window, (b) Mediastinal window).

Table 2. Lung function after one month of treatment.

\begin{tabular}{cccc}
\hline Lung function & Predictive value & Actual Value & percent $\%$ \\
\hline VC (L) & 3.16 & 2.09 & 66 \\
FEV1 (L) & 2.46 & 1.55 & 63 \\
FEV1/FVC (\%) & & 47.67 & \\
\hline
\end{tabular}

polyps are even rarer [5] [6]. The most frequent clinical symptoms are recurrent respiratory infections, obstructive pneumonia and recurrent hemopthysis [1] [7]. Although the pathogenesis of fibroepithelial polyps remains unresolved, chronic inflammatory processes may play a key role. $\mathrm{EH}$ is a specific form of the intra-pulmonary hamartoma which originates from a large bronchus, grows into the lumen and causes bronchial obstruction [8]. They constitute between 8 and $20 \%$ of all lung hamartomas [7] [8] [9]. Male predominance has been described [1] [10] with a male/female ratio of $6: 1$ and a mean age of $60-70$ years [1]. The majority $(>80 \%)$ of patients with hamartomas are smokers [10]. Computed tomography of the chest can aid in the diagnosis of endobronchial hamartoma either indirectly, by revealing the presence of obstructive pneumonia or atelectasis [11], or directly, by showing an endobronchial lesion with fat density [12] [13]. Histopathological examination is considered the gold standard for proving the diagnosis. The pathological diagnosis depends on the coexistence of different mesenchymal tissues like epithelium, connective tissue, bone, cartilage, muscle and fat [12] [14]. In our case, because of COPD, the preliminary shortness of breath of benign lung tumor was easy to be ignored by physician. Histopathology from the first biopsy only showed the ciliated columnar epithelium and squamous epithelium covered with clear cell morphology without any hamartoma pathological characteristic. After cutting, the lesion was almost moved; therefore it was abundant to diagnose hamartoma by pathology.

There are many ways to treat endobronchial hamartomas. However, endoscopically and surgically are the most common and effective methods. Treatment varies according to the size, activity and hardness of the lesion. Because of less 
severer complications, there is increasing case reports and case series using bronchoscopic [15]. Cryotherapy is the application of extreme cold energy to diseased tissue. The lesion can be destroyed by the formation of intracellular ice crystals. The first case was reported in 1968 [16] [17].

Endobronchial laser resection and cryotherapy can be used especially in patients who either refuse to have an operation or are not good surgical candidates. Surgical therapy, by bronchotomy or resection, should be reserved only for cases where the hamartomas cannot be approached through endoscopy. We herein reported a successfully treated an endobronchial hamartoma case with COPD by resection using a snare electrocautery probe and cryotherapy.

\section{Conclusion}

In conclusion, endobronchial hamartomas are one of benign neoplasms. It can cause persistent bronchial obstruction and recurrent pneumonias. The manifestations of EH are easy to be ignored in those with Chronic airway disease, such as COPD. Invasive options of bronchoscopic treatment such as forceps biopsy, electrosurgical snare, argon plasma coagulation or endobronchial laser therapy can be chosen according to the characters of leision, the presence of adjacent vessels and the wishes of patient. In this case, invasive endoscopic treatment provides an excellent outcome.

\section{Funding}

This study was supported by Shanghai Municipal Science and Technology Commission Research Project (14411951100). The projects funding receiver is Xiaodong Chen.

\section{Conflicts of Interest}

There is no conflict of interest.

\section{References}

[1] Cosío, B.G., Villena, V., Echave-Sustaeta, J., de Miguel, E., Alfaro, J., Hernandez, L. and Sotelo, T. (2002) Endobronchial Hamartoma. Chest, 122, 202-205. https://doi.org/10.1378/chest.122.1.202

[2] Kim, M.H., Lee, K.H., Kim, K.U., Park, H.K., Kim, Y.D., Lee, M.K. and Park, S.K. (2011) Patient with Positional Wheezing Due to Endobronchial Lipomatous Hamartoma. The Journal of Thoracic and Cardiovascular Surgery, 59, 188-190. https://doi.org/10.1055/s-0030-1250428

[3] Radosavljevic, V., Gardijan, V., Brajkovic, M. and Andric, Z. (2012) Lung Hamartoma-Diagnosis and Treatment. Medical Archives, 66, 281-282. https://doi.org/10.5455/medarh.2012.66.281-282

[4] Cetinkaya, E., Gunluoglu, G., Eyhan, S., Gunluoglu, M.Z. and Dincer, S.I. (2011) A Hamartoma Located in the Trachea. Annals of Thoracic and Cardiovascular Surgery, 17, 504-506. https://doi.org/10.5761/atcs.cr.10.01608

[5] Murray, J., Kielkowski, D. and Leiman, G. (1991) The Prevalence and Age Distribution of Peripheral Pulmonary Hamartoma in Adult Males. An Autopsy Based Study. 
South African Medical Journal, 79, 247-249.

[6] Li, M.I., Zhang, G., Peng, A. and Wang, C. (2013) Bronchial Fibroepithelial Polyp: A Case Report and Review of the Literature. Internal Medicine, 52, 373-376. https://doi.org/10.2169/internalmedicine.52.8864

[7] Le Roux, B.T. (1964) Pulmonary Hamartoma. Thorax, 19, 236-243. https://doi.org/10.1136/thx.19.3.236

[8] Ucar, N., Akpinar, S., Aktas, Z., Sipit, T. and Ozaydin, E. (2014) Resection of Endobronchial Hamartoma Causing Recurrent Hemoptysis by Electro-Cautery and Cryotherapy. Hippokratia, 18, 355-356.

[9] Tomashefski, J.F. (1982) Benign Endobronchial Mesenchymal Tumors: Their Relationship to Parenchymal Pulmonary Hamartomas. The American Journal of Pathology, 6, 531-540. https://doi.org/10.1097/00000478-198209000-00005

[10] Gjevre, J.A., Myers, J.L. and Prakash, U.B. (1996) Pulmonary Hamartomas. Mayo Clinic Proceedings, 71, 14-20. https://doi.org/10.4065/71.1.14

[11] Altin, S., Dalar, L., Karasulu, L., Çetinkaya, E., Timur, S. and Solmazer, N. (2007) Resection of Giant Endobronchial Hamartoma by Electrocautery and Cryotherapy via Flexible Bronchoscopy. Tuberk Toraks, 55, 390-394.

[12] Stey, C.A., Vogt, P. and Russi, E.W. (1998) Endobronchial Lipomatous Amartoma. A Rare Cause of Bronchial Occlusion. Chest, 13, 254-255. https://doi.org/10.1378/chest.113.1.254

[13] Ahn, J.M., Im, J.G., Seo, J.W., Han, H.S., Yoon, H.K., Kim, W.S. and Yeon, K.M. (1994) Endobronchial Hamartoma: CT Findings in Three Patients. AJR, 163, 49-50. https://doi.org/10.2214/ajr.163.1.8010245

[14] Borro, J.M., Moya, J. and Botella, A. (1989) Endobronchial Hamartoma, Report of Seven Cases. Scandinavian Journal of Thoracic and Cardiovascular Surgery, 23, 285-287. https://doi.org/10.3109/14017438909106011

[15] Sim, J.K., et al. (2014) Two Cases of Diagnosis and Removal of Endobronchial Hamartoma by Cryotherapy via Flexible Bronchoscopy. Tuberculosis and Respiratory Diseases, 76, 141-145. https://doi.org/10.4046/trd.2014.76.3.141

[16] Sunna, R. (2013) Cryotherapy and Cryodebridement. In: Ernst, A. and Herth, F.J., Eds., Principles and Practice of Interventional Pulmonology, Springer Science, New York, 343-350. https://doi.org/10.1007/978-1-4614-4292-9_33

[17] Morgan, R.K. and Ernst, A. (2010) Cryotherapy. In: Strausz, J. and Bolliger, C.T., Eds., Interventional Pulmonology: European Respiratory Monograph, European Respiratory Society, Sheffield, 161-172. https://doi.org/10.1183/1025448x.00991209 